\title{
A re-audit of the delivery of dietitian-prescribed snacks and oral nutritional supplements to elderly inpatients
}

\author{
S. Kaur ${ }^{1}$, Y. Jeanes ${ }^{1}$ and C. E. Weekes ${ }^{2}$ \\ ${ }^{1}$ School of Human and Life Sciences, Roehampton University, London SW15 5JD, UK and ${ }^{2}$ Department of Nutrition \\ and Dietetics, Guy's \& St Thomas' NHS Foundation Trust, Westminster Bridge Road, London SE1 7EH, UK
}

A recent audit ${ }^{(1)}$ showed only approximately $25 \%$ snacks and approximately $10 \%$ oral nutritional supplements (ONS) were delivered to the correct patient at the suggested time on the elderly care wards of an acute hospital Trust (audit 1; spring 2006). Following the audit a number of strategies were implemented, including weekly meetings between dietitians and diet chef, liaison with staff in the catering loading bay and training for ward staff and dietitians. Dietitians were also trained to write ONS prescriptions on drug charts. The aim of the current study was to determine if these strategies resulted in improved delivery of dietitian-prescribed snacks and ONS.

The study (audit 2; summer 2007) was conducted on four elderly care wards over a 6-week period. During the initial 4 weeks the following data were recorded for all elderly inpatients identified as requiring snacks and ONS: number of dietitian-prescribed items; number of correctly labelled items that arrived on the ward; number of items offered to the correct patient at the suggested time. A qualitative study was conducted over the following 2 weeks to establish reasons why the correct snacks and ONS failed to arrive with the correct patient at the suggested time.

\begin{tabular}{|c|c|c|c|c|c|c|c|c|c|c|}
\hline & \multicolumn{2}{|c|}{ Patients who reveived } & \multicolumn{4}{|c|}{ Items prescribed } & \multicolumn{4}{|c|}{$\begin{array}{l}\text { Items offered to correct patient } \\
\text { at suggested time }\end{array}$} \\
\hline & \multirow{2}{*}{$\begin{array}{c}\text { Audit } 1 \\
n 31\end{array}$} & \multirow{2}{*}{$\begin{array}{c}\text { Audit } 2 \\
n 35\end{array}$} & \multicolumn{2}{|c|}{ Audit 1} & \multicolumn{2}{|c|}{ Audit 2} & \multicolumn{2}{|c|}{ Audit 1} & \multicolumn{2}{|c|}{ Audit 2} \\
\hline & & & $n$ & $\%$ & $n$ & $\%$ & $n$ & $\%$ & $n$ & $\%$ \\
\hline Snacks & 1 & 11 & 329 & 52 & 131 & 44 & 89 & 27 & 88 & 67 \\
\hline ONS & 8 & 19 & \multirow{2}{*}{\multicolumn{2}{|c|}{302}} & 165 & 56 & 27 & 9 & 87 & 53 \\
\hline Snacks + ONS & 22 & 5 & & & \multicolumn{2}{|c|}{ - } & \multicolumn{2}{|c|}{ - } & \multicolumn{2}{|c|}{ - } \\
\hline
\end{tabular}

A significant improvement was observed in the proportion of correctly labelled snacks being delivered to the wards from the catering loading bay (audit $1, n 196(60 \%)$; audit $2 n 112(85 \%) ; P<0.01)$. Once on the ward a greater proportion of snacks $(P<0.01)$ and ONS $(P<0.01)$ were offered to the correct patient at the suggested time (see Table). During audit 2 the main reason that patients failed to receive ONS was when the prescription was not written on the drug chart $(n 44 ; 27 \%)$, since dietitians new to the Trust were required to record ONS on the end-of-bed chart until they were trained to write on the drug chart. The main reason that patients failed to receive the correct snacks was inadequate communication on the ward.

A significant reduction was observed in the median number of snacks (audit 1,8 (range 0-39); audit 2, 0 (range 0-25); $P=0.003$ ) and ONS prescribed per patient (audit 1,8 (range 0-26); audit 2, 5 (range $0-15$ ); $P=0.001$ ). This result may reflect a change of dietetic practice between the two audits, although it should be noted that the mean length of stay on the elderly care wards decreased between audit 1 and audit 2 from $20 \mathrm{~d}$ to $15 \mathrm{~d}$ and the audits took place at different times of the year.

Staff training and improved liaison resulted in significant improvements in the delivery of dietitian-prescribed snacks and ONS. However, approximately $50 \%$ patients failed to receive the correct ONS and $>30 \%$ failed to receive the correct snacks at the suggested time.

1. Bremang MP, Callow LJ \& Weekes CE (2007) Proc Nutr Soc 66, 4A. 